\title{
DOI 10.31489/2021No1/34-42 \\ UDC 621.313.333.07:621.315.616.9 \\ IMPROVING THE HEAT RESISTANCE OF POLYMER ELECTRICAL INSULATION SYSTEMS FOR THE MODERNIZATION OF INDUCTION MOTORS
}

\author{
Leonov A. ${ }^{1}$, Usacheva T. ${ }^{1}$, Lyapunov D. ${ }^{1}$, Voronina N. ${ }^{1}$, Galtseva O. ${ }^{2}$, Rogachev A. ${ }^{3}$ \\ ${ }^{1}$ National Research Tomsk Polytechnic University, Tomsk, Russia, voronina@tpu.ru \\ 2Tomsk State University of Control Systems and Radioelectronics, Tomsk, Russia \\ ${ }^{3}$ Belarusian State University of Transport, Gomel, Belarus
}

\begin{abstract}
Ensuring high power, efficiency and reliability of induction motor operation while providing small weightsize parameters represents an up-to-date problem. One of the factors limiting the reduction of weight-size parameters when increasing capacities of induction motors is the failure of the insulation system. Therefore, there is a strong need for studying the issues of slot fill when designing an induction motor, taking into account an increase in the heat resistance class of electrical insulation systems. The purpose of the present research is to develop a polymeric electrical insulation system of increased heat resistance for the modernization of an induction medium-capacity motor to reduce weight-size parameters and to evaluate the possible savings of winding material. The paper deals with an analysis on the stator-slot fill for the induction motor. Modern conductor and insulation materials were selected. The necessary calculations for a medium-power induction motor of different heat resistance classes were performed. The usage of the proposed electrical insulation system was substantiated. The analysis shows the possibility of increasing the heat resistance of the polymer electrical insulation systems to save winding material for induction motors without reducing the specified quality level.
\end{abstract}

Keywords: electrical insulation system, polymeric materials, heat resistance, induction motor, weight-size parameters.

\section{Introduction}

The problems of ensuring high capacity, economical efficiency and reliability of induction motor operation while ensuring small weight-size parameters are among the most acute problems associated with the development of modern electrical engineering [1]. The primary purpose of induction motors modernization lies in energy performance enhancement [2]. At the same time, issues of material saving have faded into the background. At present, the issues of increasing capacity and reducing the size-weight parameters of electrical devices are often solved simultaneously [3 - 6]. Among the factors limiting the reduction of weight-size parameters while increasing the induction motor capacities is the failure of the insulation system [7-11]. In this case, under the insulation system of an induction motor we mean the stator winding and its various elements. In addition to the winding, the machine slot is filled with the case insulation, turn separator and conductor insulation as well as various insulating spacers.

During the motor operation, the currents flow through the windings causing the insulation heating leading to its aging. Thus, the insulation lifetime and, consequently, the entire electrical device lifetime are sharply reduced [12 - 14]. To ensure the reliability of the motor operation $[15,16]$, the motor insulation must provide the following requirements:

1) high electric strength, which prevents turn-to-turn short-circuit and winding short-circuit with metal parts of the electric motor;

2) high thermal conductivity, that increases the efficiency of heat transfer to the environment to prevent insulation overheating.

The motor weight-size parameters are largely determined by the thickness of the inter-turn and slot insulation. The thickness and design of the insulation is determined by its purpose and type, by the level of the rated voltage, and motor operating conditions [17 - 19]. On the other hand, the large insulation thickness determines the reliability of the electric motor. To increase the heat resistance of polymer electrical insulation systems we need to employ the trend of miniaturization in the production of ultra-thin enameled wires. Therefore, to reduce the weigh-size parameters of an induction motor we need to investigate deeper the problem of stator slot filling using polymer insulators with a higher class of heat resistance. 


\section{Winding and Insulation Materials for Medium Power Induction Motors}

Conventionally, the stator windings of medium-power induction motors are made of copper wires. The durability of the winding wires determine safe motor operation throughout the life cycle. The durability depends on a bunch of the wires' properties. However, the heat resistance was chosen throughout the world as the most important characteristic of electrical and insulation materials. This characteristic is directly related to a long-term permissible operating temperature [20].

Belonging to one or another class of heat resistance determines the insulation properties. The heat resistance class of enamel insulation of wires depends on the chemical composition of enamel varnish. Electrical insulating varnishes represent solutions of high molecular weight film-forming compounds or low molecular weight reactive oligomers in organic volatile liquids. When the varnish is heated in a furnace, the molecular weight of the film-forming compounds increases and the solvent evaporates. Finally, a solid enamel film forms on the wire. Some vegetable oils and synthetic resins are used as film-forming agents. Synthetic varnishes form more stable and heat-resistant enamel films on the wire.

For the production of enameled wires of heat resistance class $\mathrm{B}\left(130^{\circ} \mathrm{C}\right), \mathrm{F}\left(150^{\circ} \mathrm{C}\right), \mathrm{H}\left(180^{\circ} \mathrm{C}\right)$ manufacturers use varnishes on polyester, polyetherimide and polyamide bases. Heat resistance improvement of polymer electrical insulation systems will increase the heat resistance class of electrical devices with the same geometry of the winding wire. Consequently, this will lead to a rise in the slot fill factor of induction motors. Therefore, the power of the motors will rise, and weight-size parameters will drop significantly. These outcomes allow recommend employing the winding wire of a heat resistance class of $\mathrm{H}$ as a perspective way to manufacture a winding insulation system for induction motors. Thus, while improving the induction motors, the insulation thickness remains unchanged. However, the winding wires enamel will be the subject to change during fabrication. Moreover, the required heat resistance class of the windings is expected to strongly depend on the heat resistance class.

The conducted review of winding materials shows the relevance of using the copper winding wires to increase the heat resistance of polymer electrical insulation systems when developing an insulation system for medium-power induction motors with the heat resistance of class F. The cross-section the conductor wire is $1.767 \mathrm{~mm}^{2}$. For the slot insulation system with heat resistance of class $\mathrm{N}$, a copper winding wire with a cross-section of $1.539 \mathrm{~mm}^{2}$ was chosen. Both wires are enameled with polyesterimide varnish.

Since we improve the heat resistance class of the windings, we also need to choose more heat resistant materials for the case and slot insulation. The insulation thickness is determined by the characteristics of the insulating material, such as heat resistance, dielectric strength, and others. The thickness affects to a greater extent the geometric dimensions of the slots and their filling. Limiting the size of the slot makes it necessary to design slot insulation in the form of a thin layer having high mechanical strength and corresponding to the requirements of induction motor insulation. Generally, imidoflex 292 based film-glass fiber with the thickness of $0.25 \mathrm{~mm}$ is used as the case insulation. Imidoflex 292 is an insulating glass wrapping, glued on both sides with a heat-resistant polyimide film. A characteristic feature of imidoflex is the resistance to high temperatures and maintaining the required level of performance with increasing in temperature. The heat resistance class of imidoflex is H. Sintoflex 82 and 828 have the same heat resistance class. Sintoflex 82 represents a film insulating material with high resistance to electrical, thermal and mechanical stress. This material is composed of a polyimide film (the most heat-resistant polymer material) and aramid paper on both sides, which possesses an original combination of characteristics. The only drawback of this material is its high cost, which is justified by the high operational characteristics of the material and long operating lifetime. The main technical characteristics of insulating materials discussed above are given in Table 1.

Table 1. Technical characteristics of insulating materials

\begin{tabular}{|c|c|c|c|c|}
\hline Material grade & Thickness, mm & $\begin{array}{c}\text { Surface density, } \\
{\left[\mathrm{g} / \mathrm{m}^{2}\right]}\end{array}$ & $\begin{array}{c}\text { Breakdown voltage, } \\
{[\mathrm{kV}], \text { not less than }}\end{array}$ & $\begin{array}{c}\text { Heat resistance class, } \\
{\left[{ }^{0} \mathrm{C}\right]}\end{array}$ \\
\hline Imidoflex 292 & $0.13-0.50$ & $170-892$ & 8 & 180 \\
\hline Syntoflex 82 & 0.18 & 191 & 10 & 180 \\
\hline Syntoflex 828 & $0.15-0.24$ & $170-248$ & 10 & 180 \\
\hline
\end{tabular}




\section{Change in Electrical Insulation System with Heat Resistance Increasing of Induction Motors}

The insulation system construction is located in the stator slots of the induction motor. When designing, it is reasonable to choose the smallest slot sizes that ensure the placement of the required number of wires and their insulation. The degree of the slot volume utilization characterizes by the fill factor. The margin of the stator permissible overheating can be substantially increased by choosing the proper materials for the electrical insulation system with increased heat resistance. Reducing the insulation thickness results in an improvement in heat dissipation in the active parts of the motor. Besides, this enhances the cooling conditions. This gives grounds to reduce the geometry of the slots as well as the sizes of the motor [21]. The efficiency of using active materials, taking into account the geometry of electric motors, is determined by electromagnetic loads being the linear load A and the magnetic flux density in the air gap B $\delta$. Permissible heating of the active parts of induction motors determines the allowable levels of electromagnetic loads, since with the growth of $\mathrm{A}$ and $\mathrm{B} \delta$ the losses per unit volume in the motor increase. Choosing large values of the linear load and the magnetic flux density in the air gives the best usage of active materials. This leads to sufficient material savings.

The ranges of permissible values of $\mathrm{A}$ and $\mathrm{B} \delta$ are determined on the basis of experience gained during the design and operation of the induction motors of various ratings. The above mentioned values ensure that the over temperature does not exceed the allowable one for the adopted class of insulation heat resistance. Implementing electrical insulation systems with increased heat resistance demands recalculating the number of turns per motor phase, the number of effective conductors in the slot and the cross-section of the stator winding wire. The number of turns in the phase of the stator winding is determined by the following relation:

$$
w=\frac{k_{e} U_{1 n}}{4 k_{v} k_{w} f \Phi}
$$

where $k_{e}$ is the ratio of the EMF of the stator winding to the rated voltage; $U_{I n}$ is rated voltage; $k_{v}$ is the field shape factor; $k_{w}$ is the winding factor; $\mathrm{f}$ is the power supply frequency; $\Phi$ is a magnetic flux.

The number of effective conductors in the slot is calculated as follows:

$$
u_{c}=\frac{\pi D A}{I_{1 n} Z_{1}}
$$

where $D$ is the inner diameter of the stator; $A$ is the previously accepted value of the linear load; $I_{I n}$ is rated current of the stator winding; $Z_{l}$ is the number of stator slots.

The cross section of the stator winding wire:

$$
q_{e f f}=\frac{I_{1 n}}{a J_{1}},
$$

where $I_{l n}$ is the rated current of the stator winding; $a$ is the number of parallel branches of the stator winding; $J_{l}$ is the current density in the stator winding.

Updating the number of the effective conductors is performed through the Equation:

$$
u_{p}^{\prime}=\frac{u_{p}}{a}
$$

where $u_{p}$ is the number of effective conductors in the slot; $a$ is the number of parallel branches of the stator winding.

The above parameter variation depends on electromagnetic loads. The total values are determined by the Equations:

$$
\begin{aligned}
& A_{\text {total }}=\frac{2 I_{1 n} w m}{\pi D}, \\
& B_{\text {dtotal }}=\frac{p \cdot \Phi}{D \cdot l_{\delta}} .
\end{aligned}
$$

where $I_{l n}$ is the rated current of the stator winding; $w$ is the number of turns in the phase of the stator winding; $m$ is the number of phases; $D$ is the inner diameter of the stator; $p$ is the number of pole pairs; $\Phi$ is magnetic flux; $l_{\delta}$ is the estimated length of the magnetic circuit. 
The relation to check the permissible current density is expressed by the following relation:

$$
J_{1}=\frac{A J}{A_{\text {total }}} \text {, }
$$

where $A J$ is the product of linear load and current density; $A_{\text {total }}$ is the final linear load value.

Figure 1 illustrates clearly the change in the geometry of the stator slot and its filling when implementing electrical insulation systems with increased heat resistance. Changing the geometry of the slot leads to a change not only in the number of turns, the sizes of the winding wire and the values of electromagnetic loads, but also in other parameters, affecting, in turn, the parameters of the operating mode of induction motors such as power loss, efficiency $\eta$ and power factor $\cos \varphi$. Therefore, the need to assess the required performance level of the improved induction motor arises. The determining factors of such an assessment are technological factors, performance indicators and allowable error band imposed on them.

As technological factors we consider the geometric sizes of the parts (e.g. slots), and requirements imposed on them in terms of accuracy in manufacturing. When designing the correctness of the proposed modernization method is evaluated by performance indicators, normalized by GOST 26772-85. For mediumpower induction motors we adopted the following performance indicators, where $\eta$ is efficiency, $\cos \varphi$ is power factor, $M_{s}$ is starting torque ratio, $I_{s}$ is starting current ratio. The performance indicators for electrical devices are given as either the accurate values or acceptable ranges of the values.

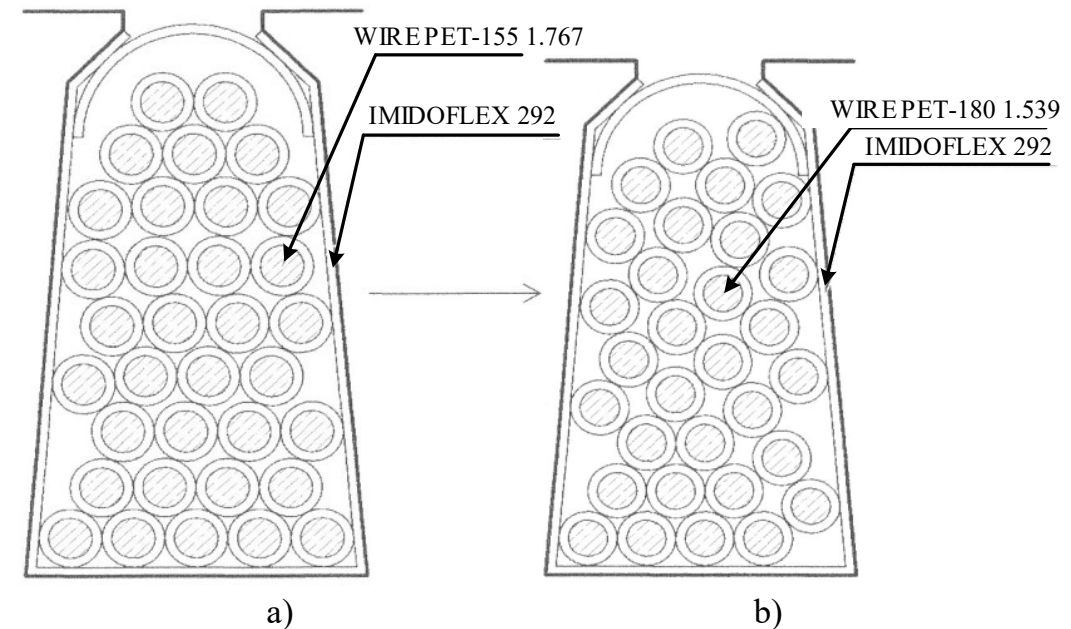

Fig.1. The change in the geometry of the stator slot and its filling for:

a) heat resistance class F; b) heat resistance class $\mathrm{H}$

The characteristic feature of the electric motors lies in the presence of one-sided error bands for the performance indicators, which expands the leverage for electrical devices modernization by taking into account the accuracy of the theoretical results adjustment and determining optimal conditions for the modernization of induction motors [22]. Changing the geometry or the stator slot fill will probably lead to a change in the thermal state of the induction motor, which can cause a decrease in the nonfailure operating time. Thus, the heat fluxes, as well as the temperature changes in the internal volume of the machine and on the cooling surfaces represent the important factors that need to be evaluated. In order to verify compliance with the requirements for the permissible heating level of an induction motor, the average temperature rise of the stator winding $\Delta_{\text {vav }}$ is determined. The valid values for this parameter are given in the specification papers. The block diagram of the induction motors modernization when changing electrical insulation systems with increased heat resistance is represented in Figure 2. The basis of the given block diagram is a set of interconnected electromagnetic and thermal calculations. This allows us to assess the performance of the designed induction motor, to evaluate its possible technical characteristics, to establish the relationship between the characteristics and the weight-size parameters, and, finally, to determine the optimal values of the parameters for successful construction modernization. To render complex processes, such as the induction motor modernization, we need to establish the relationship between the information about the input of each element in the block diagram and its output. In this case the basic data (ratings, motor sizes, electrical quantities) and auxiliary data (coefficients and per unit values of the parameters) are used as initial data. At this modernization stage, changes in electromagnetic loads were chosen as independent variables. We 
consider the opportunity of reducing the specific material consumption. Therefore, the block diagram should comprise the calculation of the mass and specific material consumption of the materials used to manufacture induction motors. The performance indicators and average over temperature of the stator windings represent the limitations in the block diagram (Fig. 2). The variation of the independent variables must be carried out until at least one of the limitation is not satisfied.

\section{Calculation of Induction Motor Parameters when Implementing Electrical Insulation System with Improved Heat Resistance}

The design of induction motors comes down to repeatedly calculating the dependencies between the performance indicators. Basically, these indicators are defined by a system of equations, empirical coefficients, graphical dependencies, which are considered as a set of equations for design. The block diagram (Fig. 2) is based on standard electromagnetic and thermal calculations. Electromagnetic calculations are used to design the manufacturing process of an induction motor, whereas thermal calculations allow to evaluate the thermal state of the machine in the course of modernization.

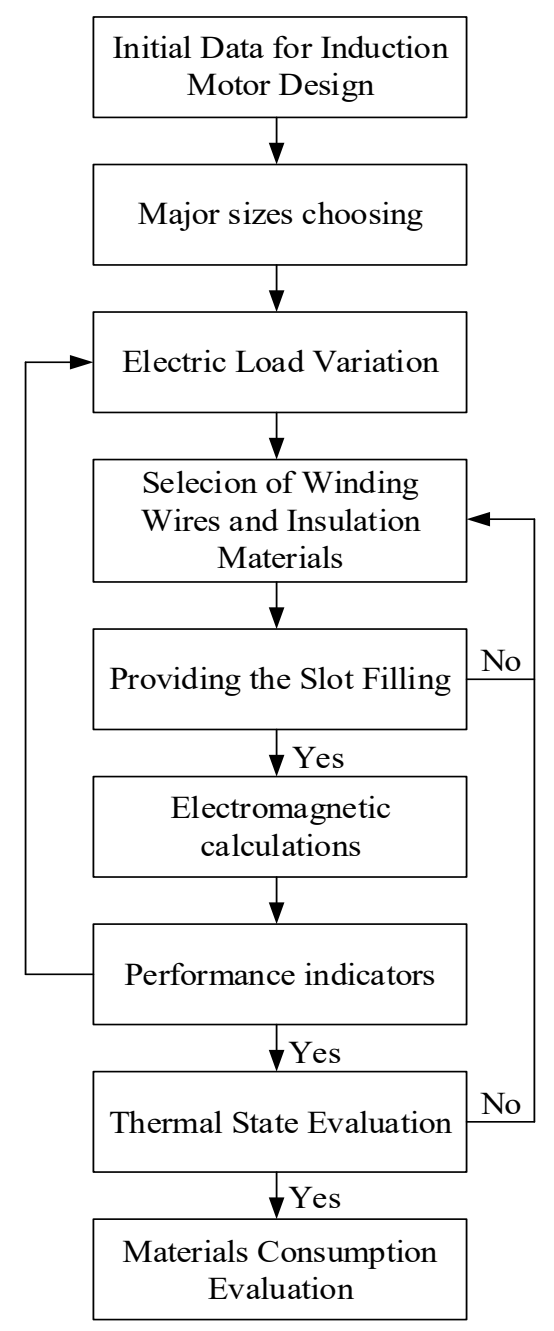

Fig.2. The block diagram of the induction motor design procedure by varying electrical insulation systems with improved heat resistance

To verify the calculations, we choose the medium-power induction motor AIR112M4 with the heat resistance class of $\mathrm{F}$. The calculation results are shown in Table 2. The table presents a comparison of the calculated values for the standard induction motor with insulation system of heat resistance of class $F$ and the improved induction motor AIR12M4 with insulation system of heat resistance of class $\mathrm{H}$ with decreased slot geometry. The calculated values of the main electrical quantities, performance indicators and the average 
temperature rise of the stator winding are given for a comparative analysis. When solving problems of reducing the geometry of the stator slot it should be noted that usually electric motors are mass-produced. When designing a line of electrical motors, the issues of the component unification and the normalization of motor sizes are of utmost importance. Reducing the height of the axis of rotation beyond the standard values will lead to changes in the geometry of the cross section of the motor.

This will entail a change in the stamps for manufacturing and will require large capital costs for foundry, stamping, machining and other equipment [23]. When changing the height of the axis of rotation from $112 \mathrm{~mm}$ to the standard value of $100 \mathrm{~mm}$ we left the geometry of the cross section of the machine unchanged (corresponding to $\mathrm{h}=100 \mathrm{~mm}$ ). To ensure the rated power for smaller sizes of the cross section, we can only change the lengths of the stator and rotor cores. The height of the axis of rotation is the main installation parameter by which the motor is coupled to the mechanism. In our case, different external diameters of the stator core will correspond to one height of the axis of rotation. It is possible to realize the unification of the stator core for different dimensions of the induction motor by increasing the arms or developing the design of the frames with removable arms. All this is only possible within the framework of mass production, since it will entail only a retooling of the equipment and changes in equipment.

Table 2. Total calculation results for induction motor AIR112M4 when utilizing electrical insulation systems with increased heat resistance

\begin{tabular}{|l|l|l|}
\hline Class & $\mathrm{F}\left(155^{\circ} \mathrm{C}\right)$ & $\mathrm{H}\left(180^{\circ} \mathrm{C}\right)$ \\
\hline$h,[\mathrm{~mm}]$ & 112 & 100 \\
\hline$P_{2 \mu},[\mathrm{W}]$ & 5500 & 5500 \\
\hline $\mathrm{Da},[\mathrm{m}]$ & 0.191 & 0.168 \\
\hline$l_{1},[\mathrm{~m}]$ & 0.125 & 0.155 \\
\hline$I_{1},[\mathrm{~A}]$ & 11.265 & 11.419 \\
\hline$\eta$ & 0.858 & 0.849 \\
\hline $\cos \varphi$ & 0.863 & 0.86 \\
\hline$M_{s}$ & 2.873 & 2.206 \\
\hline$I_{s}$ & 6.582 & 6.54 \\
\hline$\Delta v_{a v},\left[{ }^{\circ} \mathrm{C}\right]$ & 68.998 & 92.797 \\
\hline$\Delta v_{p e r},\left[{ }^{\circ} \mathrm{C}\right]$ & 92 & 112 \\
\hline
\end{tabular}

The quantitative assessment of the induction motor AIR112M4 modernization showed that the real limitation is the motor efficiency. Though, if the efficiency value takes a minimum acceptable value, the power factor value is acceptable. The average over temperature of the stator winding when decreasing the geometry parameters is below the limit value $\Delta v_{a v}=92.797^{\circ} \mathrm{C}$. Thus, the average over temperature of the stator winding is within acceptable limits and has a significant margin. This confirms the possibility of using the proposed high-temperature-resistant electrical insulation systems for induction motors in order to reduce the material consumption for electrical devices.

\section{Assessment of the Potential of Reducing Material Consumption when Using an Electrical Insulation System with Increased Heat Resistance}

One of the most important areas of increasing the competitiveness of electric machines is the reduction of material consumption, which leads to a decrease in weight - size parameters. To evaluate the motor modernization conducted, it is necessary to find a variant of the designed induction motor that ensures the performance of the specified technical functions with lower weight-size parameters. The most important criterion, from the point of view of reducing the material consumption of electric machines, is materials saving: electrical steel, copper, aluminum. We consider the minimum material consumption as an optimization criterion for constructing the target function: 


$$
f\left(l_{1}, l_{2}\right)=\left(M_{s t}-D_{a}^{2} l_{1} \gamma_{s t}\right) \cdot C_{s t}+\left(M_{C u}-\gamma_{C u} \cdot 2\left(l_{1}+l_{f}\right) w_{1} q_{e f f}\right) \cdot C_{C u}+\left(M_{A l}-\gamma_{A l}\left(Z_{2} S_{c} l_{2}+2 \pi D_{r} q_{r}\right)\right) \cdot C_{A l}
$$

where $M_{s t}$ is the mass of steel for the stator and rotor cores in mass production; $M_{C u}$ is the mass of copper stator winding; $M_{A l}$ is the mass of aluminum rotor winding in; $C_{s t}, C_{C u}, C_{A l}$ are the costs of $1 \mathrm{~kg}$ of electrical steel, copper for stator winding and aluminum for rotor winding, respectively; $\gamma_{s t}, \gamma_{C u}, \gamma_{A l}$ are specific masses of steel, copper and aluminum, respectively; $l_{f}$ is the length of the frontal part of the winding; $S_{c}$ is the cross section of the rotor slot; $D_{a}$ is the outer diameter of the stator core; $D_{r}$ is the average diameter of the closing rings; $q_{r}$ is the cross section of the closing rings.

To assess the reduction in mass without the cost of materials, it is advisable to calculate the target functions in percent separately for each type of material:

$$
\begin{aligned}
& \Delta M_{s t}=\frac{\left(M_{s t}-D_{a}^{2} l_{1} \gamma_{s t}\right)}{M_{s t}} 100 \% \\
& \Delta M_{C u}=\frac{M_{C u}-\gamma_{C u} \cdot 2\left(l_{1}+l_{f}\right) w_{1} q_{e f f}}{M_{C u}} 100 \% \\
& \Delta M_{A l}=\frac{M_{A l}-\gamma_{A l}\left(Z_{2} S_{s} l_{2}+2 \pi D_{r} q_{r}\right)}{M_{A l}} 100 \%,
\end{aligned}
$$

The potential of reducing the material consumption of induction motors is determined by calculating the reduction in the material consumption of the improved induction motors expressed in percent $(\Delta M)$ and the specific material consumption of the improved induction motors (number of materials per unit power $G$ ) for each type of material, namely steel, copper, aluminum, i.e. a quantitative assessment of the reduction in the material consumption of induction motors is given:

$$
\begin{aligned}
& \Delta M=\frac{M_{B}-M_{I}}{M_{B}} 100 \% \\
& G=\frac{M_{I}}{P_{2 n}},
\end{aligned},
$$

where $M_{B}$ is the mass of the induction motor taken as a base, $M_{I}$ is the mass of the improved induction motor.

Table 3 shows the values of the change in mass and specific material consumption of active materials for induction motor AIR112M4 with different versions of electrical insulation systems with increased heat resistance.

Table 3. Changes in mass and specific material consumption of active materials for induction motor AIR112M4 for different types of electrical insulation systems with increased heat resistance.

\begin{tabular}{|c|c|c|}
\hline Insulation class & $\mathrm{F}\left(155^{\circ} \mathrm{C}\right)$ & $\mathrm{H}\left(180^{\circ} \mathrm{C}\right)$ \\
\hline$h,[\mathrm{~mm}]$ & 112 & 100 \\
\hline$M_{s t},[\mathrm{~kg}]$ & 35.569 & 34.123 \\
\hline$M_{C u},[\mathrm{~kg}]$ & 1.356 & 1.117 \\
\hline$M_{A l},[\mathrm{~kg}]$ & 1.529 & 1.458 \\
\hline$G_{s t},[\mathrm{~kg} / \mathrm{kW}]$ & 6.467 & 6.204 \\
\hline$G_{C u},[\mathrm{~kg} / \mathrm{kW}]$ & 0.247 & 0.203 \\
\hline$G_{A l},[\mathrm{~kg} / \mathrm{kW}]$ & 0.278 & 0.265 \\
\hline
\end{tabular}

The histogram (Figure 3) presents visual representation of the change in the mass of copper for different versions of electrical insulating systems with increased heat resistance. 


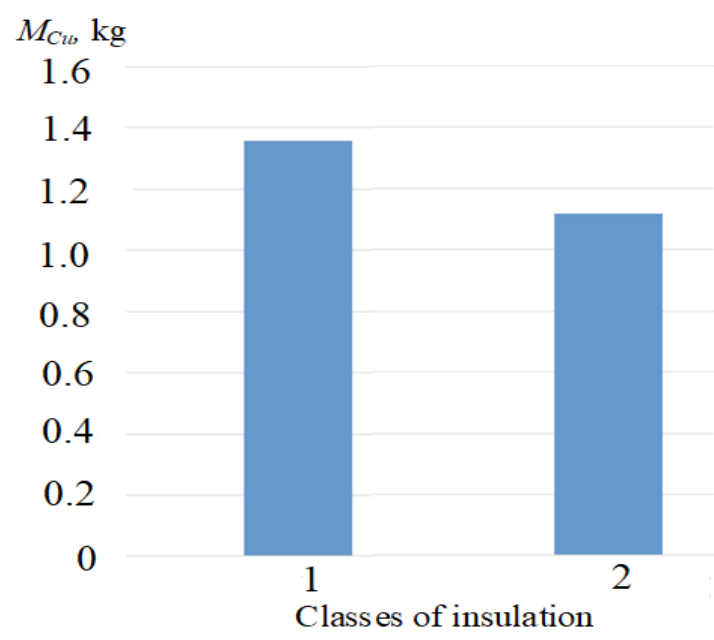

Fig.3. Change in the mass of copper in the windings of different heat resistance classes, where 1 is $\mathrm{F} ; 2$ is $\mathrm{H}$

Undoubtedly, specific material consumption is an important indicator for comparing machines of the same power ratings and different designs. This indicator characterizes the degree of materials use for the active parts of induction motors and evaluates the manufacturability of the design.

The analysis showed a clear decrease in specific material consumption for steel, copper and aluminum. Possible savings of copper winding material equals to $17.6 \%$ with respect to the base value. It should be noted that steel savings amount to $4.1 \%$. For aluminum this value is $4.6 \%$. This means that with a substantial decrease in material consumption in the course of modernization, induction motors still remain heavy in mass. Thus, when applying an electrical insulation system with increased heat resistance for induction motors, the potential of reducing the weight-size parameters and saving the electrical materials used in the manufacturing process were revealed.

\section{Conclusions}

Undoubtedly, modernization of asynchronous motors by increasing the heat resistance of polymer electrical insulating systems are urgent tasks of improving electrical products, requiring the search of optimal solutions at designing and manufacturing. These studies are the part of the complex work carried out in the School of Power Engineering of Tomsk Polytechnic University. The theory of electrical machines, the theory of the foundations of electrical insulation technology, the theory of accuracy, probability and mathematical statistics were used for solving the research tasks. These studies allowed making it possible to develop calculated algorithms and recommendations for the modernization of asynchronous motors at the design and manufacture, aimed at increasing the efficiency of asynchronous motors, namely:

1. Increasing the heat resistance of polymer electrical insulation systems can be achieved through the use of materials with improved characteristics. This makes it possible to subsequently increase the value of electromagnetic loads, which leads to a better use of active materials of induction motors and reduces material consumption by reducing the slot geometry while maintaining the capacity of the induction motor.

2. As a result of increasing the heat resistance class of the insulation system while reducing the motor sizes, it is possible to save copper for winding by $17.6 \%$ with respect to the base value.

3 . The analysis of the results shows good grounds for saving the winding material of induction motors without reducing the specified performance level applying an insulation system with increased heat resistance. Performing the optimal design of electric motors without taking into account standardization issues of transverse geometry leads to the savings of winding materials.

\section{REFERENCES}

1 Muravleva O. Resource saving prospects based on accuracy theory. Proceeding of the Intern. Conference on Computer as a Tool. In EUROCON 2007. 2007, 4400413, pp. 1845-1850, doi: 10.1109/EURCON.2007.4400413.

2 Payuk L.A., Voronina N.A., Logvinenko A.A. Modernization of electric drive of compressor station. Bulletin of the Tomsk Polytechnic University. Geo Assets Engineering. 2019, Vol. 330, No. 3, pp. 73 - 83. doi: 10.18799/24131830/2019/3/165. 
3 Muravleva O., Muravlev O. Power effective induction motors for energy saving. Proceedings of the $9^{\text {th }}$ Russian-Korean Intern. Symp. on Science and Technology KORUS-2005. 2005, Vol. 1, No. 1507731, pp. 358-362. doi: 10.1109/KORUS.2005.1507731.

4 Abramov B.I., Ivanov G.M., Leznov B.S. Power saving by electric drive means in the town municipal service. Russian Electrical Engineering. 2001, Vol. 1, pp. 6 - 11.

5 Han P.-W., Chun Y.-D., Choi J.-H., Kim M.-J., Koo D.-H., Lee J. The study to substitute aluminum for copper as a winding material in induction machine. Proceedings of INTELEC. 2009, pp. 5352008.

6 Payuk L. A., Voronina N. A., Galtseva O.V. Energy Characteristics of Electric Drive of Oscillatory Motion at the Shock-Free Start. Journal of Physics: Conference Series. 2016, Vol. 671, pp. 012044.

7 Guastavino F., Cordano D., Torello E., Secondo G. Electrical aging tests on different kind of insulating systems adopted for induction stator motor. Proceedings of Conference of CEIDP. 2015, pp. 7352068. doi: 10.1109/CEIDP.2015.7352068.

8 Bonnett A.H., Soukup G.C. Cause and analysis of stator and rotor failures in three-phase squirrel-cage induction motors, IEEE Transactions on Industry Applications. 1992, Vol. 28, No. 4, pp. 921-937, doi: $10.1109 / 28.148460$.

9 Chapman M., Frost N. Insulation systems for rotating low-voltage machines. IEEE International Symposium on Electrical Insulation. 2008, 4570323. doi: 10.1109/ELINSL.2008.4570323.

10 Hwang D., Lee K., Kim Y., Lee I., Lim T., Kim D. Accessing the insulation characteristics for stator windings of low-voltage induction motors for adjustable-speed drive applications. IEEE Industry Application Conference. 2003, Vol. 1, pp. 432-438.

11 Rehder R.H., Draper R.E., Moore B.J. How Good is Your Motor Insulation System. IEEE Electrical Insulation Magazine. 1996, Vol. 12, No. 4, pp. 8-14, doi: 10.1109/57.526942.

12 Fabiani D., Montanari G.C., Contin A. Aging acceleration of insulating materials for electrical machine windings supplied by PWM in the presence and in the absence of partial discharges. IEEE $7^{\text {th }}$ Intern. Conference on Solid Dielectrics. 2001, pp. 283-286.

13 Fenger M., Campbell S.R., Pedersen J. Motor winding problems caused by inverter drives. IEEE Industry Applications Magazine. 2003, Vol. 9, No. 4, pp. 22-31, doi: 10.1109/MIA.2003.1206913.

14 Frost N., Chapman M., Bruetsch R. Considerations for rotating low-voltage machine insulation designs. IEEE Insulation. 2008, 4570397, doi: 10.1109/ELINSL.2008.4570397.

15 Shevchuk V.P., Muravlev O. P. The estimation of technical state and relibiality of electric machines in the process of operation. Proceeding of MTT. 2008, 4897500, pp. 73 - 76. doi: 10.1109/SPCMTT.2008.4897500.

16 Leuzzi R., Monopoly V.G., Rovere L., Cupertino F., Zanchetta P. Analysis and Detection of Electrical Aging Effects on High-Speed Motor Insulation. IEEE Transactions on Industry Application. 2019, pp. $1-8$.

17 Grubic S., Aller J.M., Lu B., Habetler T.G. A survey on testing and monitoring methods for stator insulation systems of low-voltage induction machines focusing on turn insulation problems. IEEE Transactions on Industrial Electronics. 2008, Vol. 55, No. 12, pp. 4127 - 4134. doi: 10.1109/TIE.2008.2004665.

18 Lahoud N., Faucher J., Malec D., Maussion P. Electrical aging of the insulation of low voltage machines: model definition and test with the design of experiments. IEEE Transactions on Industrial Electronics. 2013, Vol. 60, No. 9, pp. 4147 - 4155. doi: 1109/TIE.2013.2245615.

19 Schemmel F., Bauer K., Kaufhold M. Reliability and statistical lifetime-prognosis of motor winding insulation in low-voltage power drive systems. IEEE Electrical Insulation Magazine. 2009, Vol. 25, No. 4, pp. 6 - 13.

20 Bohm F.R., Nagel K., Schindler H. A new generation of wire enamel for the production of magnet wires with outstanding corona resistance. Electrical Insulation and Electrical Manufacturing and Coil Winding Technology Conference. 2003, pp. 109 - 113.

21 Gedzurs A. Temperature protection methods of induction motor. Research for Rural Development, 21st International Scientific Conference Research for Rural Development. 2015, Vol. 1, pp. 258 - 263.

22 Ionel M., Stan M.-F., Virjoghe E.-O., Ionel O.-M. Algorithm for exact determination of three-phase induction machine parameters. Proceeding of International Conference on Systems. 2010, Vol. 1, pp. 636-643.

23 Boglietti A., Cavagnino A., Lazzari M., Vaschetto S. Preliminary induction motor electromagnetic sizing based on a geometrical approach. IET Electric Power Applications. 2012, Vol. 6, No. 9, pp. 583-592.

Article accepted for publication 12.10 .2020 